\title{
Diagnostic criteria and severity assessment of acute cholecystitis: Tokyo Guidelines
}

\author{
Masahiko Hirota ${ }^{1}$, Tadahiro Takada 2 , Yoshifumi Kawarada ${ }^{3}$, Yuji Nimura ${ }^{4}$, Fumihiko Miura 2 , \\ Koichi Hirata ${ }^{5}$, Toshihiko Mayumi ${ }^{6}$, Masahiro Yoshida ${ }^{2}$, Stevenen Strasberg ${ }^{7}$, Henry Pitt $^{8}$, Thomas R Gadacz ${ }^{9}$, \\ Eduardo de Santibanes ${ }^{10}$, Dirk J. Gouma ${ }^{11}$, Joseph S. Solomkin ${ }^{12}$, Jacques Belghiti ${ }^{13}$, Horst Neuhaus ${ }^{14}$, \\ Markus W. Büchler ${ }^{15}$, Sheung-Tat Fan ${ }^{16}$, Chen-Guo Ker ${ }^{17}$, Robert T. Padbury ${ }^{18}$, Kui-Hin Liau ${ }^{19}$, \\ Serafin C. Hilvano ${ }^{20}$, Giulio Belli ${ }^{21}$, John A. Windsor ${ }^{22}$, and Christos Dervenis ${ }^{23}$ \\ ${ }^{1}$ Department of Gastroenterological Surgery, Kumamoto University Graduate School of Medical Sciences, 1-1-1 Honjo, \\ Kumamoto 860-8556, Japan \\ ${ }^{2}$ Department of Surgery, Teikyo University School of Medicine, Tokyo, Japan \\ ${ }^{3}$ Mie University School of Medicine, Mie, Japan \\ ${ }^{4}$ Division of Surgical Oncology, Department of Surgery, Nagoya University Graduate School of Medicine, Nagoya, Japan \\ ${ }^{5}$ First Department of Surgery, Sapporo Medical University School of Medicine, Sapporo, Japan \\ ${ }^{6}$ Department of Emergency Medicine and Critical Care, Nagoya University School of Medicine, Nagoya, Japan \\ ${ }^{7}$ Department of Surgery, Indiana University School of Medicine, Indianapolis, USA \\ ${ }^{8}$ Department of Surgery, Washington University in St Louis and Barnes-Jewish Hospital, St Louis, USA \\ ${ }^{9}$ Department of Gastrointestinal Surgery, Medical College of Georgia, Georgia, USA \\ ${ }^{10}$ Department of Surgery, University of Buenos Aires, Buenos Aires, Argentina \\ ${ }^{11}$ Department of Surgery, Academic Medical Center, Amsterdam, The Netherlands \\ ${ }^{12}$ Department of Surgery, Division of Trauma and Critical Care, University of Cincinnati College of Medicine, Cincinnati, USA \\ ${ }^{13}$ Department of Digestive Surgery and Transplantation, Hospital Beaujon, Clichy, France \\ ${ }^{14}$ Department of Internal Medicine, Evangelisches Krankenhaus Düsseldorf, Düsseldorf, Germany \\ ${ }^{15}$ Department of Surgery, University of Heidelberg, Heidelberg, Germany \\ ${ }^{16}$ Department of Surgery, The University of Hong Kong, Hong Kong, China \\ ${ }^{17}$ Division of HPB Surgery, Yuan's General Hospital, Taoyuan, Taiwan \\ ${ }^{18}$ Division of Surgical and Specialty Services, Flinders Medical Centre, Adelaide, Australia \\ ${ }^{19}$ Department of Surgery, Tan Tock Seng Hospital / Hepatobiliary Surgery, Medical Centre, Singapore, Singapore \\ ${ }^{20}$ Department of Surgery, Philippine General Hospital, University of the Philippines, Manila, Philippines \\ ${ }^{21}$ Department of General and HPB Surgery, Loreto Nuovo Hospital, Naples, Italy \\ ${ }^{22}$ Department of Surgery, The University of Auckland, Auckland, New Zealand \\ ${ }^{23}$ First Department of Surgery, Agia Olga Hospital, Athens, Greece
}

\begin{abstract}
The aim of this article is to propose new criteria for the diagnosis and severity assessment of acute cholecystitis, based on a systematic review of the literature and a consensus of experts. A working group reviewed articles with regard to the diagnosis and treatment of acute cholecystitis and extracted the best current available evidence. In addition to the evidence and face-to-face discussions, domestic consensus meetings were held by the experts in order to assess the results. A provisional outcome statement regarding the diagnostic criteria and criteria for severity assessment was discussed and finalized during an International Consensus Meeting held in Tokyo 2006. Patients exhibiting one of the local signs of inflammation, such as Murphy's sign, or a mass, pain or tenderness in the right upper quadrant, as well as one of the systemic signs of inflammation, such as fever, elevated white blood cell count, and elevated C-reactive protein level, are diagnosed as having acute cholecystitis. Patients in whom suspected clinical findings are confirmed by diagnostic imaging are also diagnosed with acute cholecystitis. The severity of acute cho-
\end{abstract}

Offprint requests to: M. Hirota

Received: May 31, 2006 / Accepted: August 6, 2006 lecystitis is classified into three grades, mild (grade I), moderate (grade II), and severe (grade III). Grade I (mild acute cholecystitis) is defined as acute cholecystitis in a patient with no organ dysfunction and limited disease in the gallbladder, making cholecystectomy a low-risk procedure. Grade II (moderate acute cholecystitis) is associated with no organ dysfunction but there is extensive disease in the gallbladder, resulting in difficulty in safely performing a cholecystectomy. Grade II disease is usually characterized by an elevated white blood cell count; a palpable, tender mass in the right upper abdominal quadrant; disease duration of more than $72 \mathrm{~h}$; and imaging studies indicating significant inflammatory changes in the gallbladder. Grade III (severe acute cholecystitis) is defined as acute cholecystitis with organ dysfunction.

Key words Acute cholecystitis · Diagnosis · Severity of illness index $\cdot$ Guidelines $\cdot$ Infection

\section{Introduction}

Early diagnosis of acute cholecystitis allows prompt treatment and reduces both mortality and morbidity. 
The accurate diagnosis of typical as well as atypical cases of acute cholecystitis requires specific diagnostic criteria. Acute cholecystitis has a better prognosis than acute cholangitis, but may require immediate management, especially in patients with torsion of the gallbladder and emphysematous, gangrenous, or suppurative cholecystitis. The lack of standard criteria for diagnosis and severity assessment is reflected by the wide range of reported mortality rates in the literature, and this lack makes it impossible to provide standardized optimal treatment guidelines for patients. In these Guidelines we propose specific criteria for the diagnosis and severity assessment of acute cholecystitis, based on the best available evidence and the experts' consensus achieved at the International Consensus Meeting for the Management of Acute Cholecystitis and Cholangitis, held on April 1-2, 2006, in Tokyo.

\section{Diagnostic criteria for acute cholecystitis}

Diagnosis is the starting point of the management of acute cholecystitis, and prompt and timely diagnosis should lead to early treatment and lower mortality and morbidity. Specific diagnostic criteria are necessary to accurately diagnose typical, as well as atypical cases. The Guidelines propose diagnostic criteria for acute cholecystitis (Table 1). C-reactive protein (CRP) is not

Table 1. Diagnostic criteria for acute cholecystitis

A. Local signs of inflammation etc.:

(1) Murphy's sign, (2) RUQ mass/pain/tenderness

B. Systemic signs of inflammation etc.:

(1) Fever, (2) elevated CRP, (3) elevated WBC count

C. Imaging findings: imaging findings characteristic of acute cholecystitis

Definite diagnosis

(1) One item in A and one item in B are positive

(2) C confirms the diagnosis when acute cholecystitis is suspected clinically

Note: acute hepatitis, other acute abdominal diseases, and chronic cholecystitis should be excluded commonly measured in many countries. However, because acute cholecystitis is usually associatied with an elevation of CRP level by $3 \mathrm{mg} / \mathrm{dl}$ or more, CRP was included. Diagnosis of acute cholecystitis by elevation of CRP level ( $3 \mathrm{mg} / \mathrm{dl}$ or more), with ultrasonographic findings suggesting acute cholecystitis, has a sensitivity of $97 \%$, specificity of $76 \%$, and positive predictive value of $95 \%$ (level $1 \mathrm{~b}) .{ }^{1}$ After the discussion during the Tokyo International Consensus Meeting, almost unanimous agreement was achieved on the criteria (Table 2). However, $19 \%$ of the panelists from abroad expressed the necessity for minor modifications, because, in the provisional version, the diagnostic criteria did not include technetium hepatobiliery iminodiacetic acid (TcHIDA) scan as an item.

\section{Imaging findings of acute cholecystitis}

Ultrasonography findings (level 4) $)^{2-5}$

Sonographic Murphy sign (tenderness elicited by pressing the gallbladder with the ultrasound probe)

Thickened gallbladder wall $(>4 \mathrm{~mm}$; if the patient does not have chronic liver disease and/or ascites or right heart failure)

Enlarged gallbladder (long axis diameter $>8 \mathrm{~cm}$, short axis diameter $>4 \mathrm{~cm}$ )

Incarcerated gallstone, debris echo, pericholecystic fluid collection

Sonolucent layer in the gallbladder wall, striated intramural lucencies, and Doppler signals.

Magnetic resonance imaging (MRI) findings

(level 1b-4) ${ }^{6-9}$

Pericholecystic high signal

Enlarged gallbladder

Thickened gallbladder wall.

Computed tomography $(C T)$ findings (level $3 b)^{10}$

Thickened gallbladder wall

Pericholecystic fluid collection

Enlarged gallbladder

Linear high-density areas in the pericholecystic fat tissue.

Table 2. Answer pad responses on the diagnostic criteria for acute cholecystitis

\begin{tabular}{|c|c|c|c|}
\hline & Agree & $\begin{array}{c}\text { Agree, but needs } \\
\text { minor } \\
\text { modifications }\end{array}$ & Disagree \\
\hline Total $(n=110)$ & $92 \%$ & $8 \%$ & $0 \%$ \\
\hline Panelists from abroad $(n=21)$ & $81 \%$ & $19 \%$ & $0 \%$ \\
\hline Japanese panelists $(n=20)$ & $100 \%$ & $0 \%$ & $0 \%$ \\
\hline Audience $(n=69)$ & $93 \%$ & $7 \%$ & $0 \%$ \\
\hline
\end{tabular}


Tc-HIDA scans (level 4) $)^{11,12}$

Non-visualized gallbladder with normal uptake and excretion of radioactivity

Rim sign (augmentation of radioactivity around the gallbladder fossa).

\section{Severity assessment criteria of acute cholecystitis}

\section{Concept of severity grading of acute cholecystitis}

Patients with acute cholecystitis may present with a spectrum of disease stages ranging from a mild, self-limited illness to a fulminant, potentially lifethreatening illness. In these Guidelines we classify the severity of acute cholecystitis into the following three categories: "mild (grade I)", "moderate (grade II)", and "severe (grade III)". A category for the most severe grade of acute cholecystitis is needed because this grade requires intensive care and urgent treatment (operation and/or drainage) to save the patient's life. However, the vast majority of patients present with less severe forms of the disease. In these patients, the major practical question regarding management is whether it is advisable to perform cholecystectomy at the time of presentation in the acute phase or whether other strategies of management should be chosen during the acute phase, followed by an interval cholecystectomy. Therefore, to guide the clinician, the severity grading includes a "moderate" group based on criteria predicting when conditions might be unfavorable for cholecystectomy in the acute phase (level 2b-4). ${ }^{13-18}$ Patients who fall neither into the severe nor the moderate group form the majority of patients with this disease; their disease is suitable for management by cholecystectomy in the acute phase, if comorbidities are not a factor. Definitions of the three grades are given below.

\section{Mild (grade I) acute cholecystitis}

Mild acute cholecystitis occurs in a patient in whom there are no findings of organ dysfunction, and there is mild disease in the gallbladder, allowing for cholecystectomy to be performed as a safe and low-risk procedure. These patients do not have a severity index that meets the criteria for "moderate (grade II)" or "severe (grade III)" acute cholecystitis.

\section{Moderate (grade II) acute cholecystitis}

In moderate acute cholecystitis, the degree of acute inflammation is likely to be associated with increased operative difficulty to perform a cholecystectomy (level $2 b-4) \cdot{ }^{13-18}$

\section{Severe (grade III) acute cholecystitis}

Severe acute cholecystitis is associated with organ dysfunction.

\section{Criteria for the severity assessment of acute cholecystitis}

Acute cholecystitis has a better outcome/prognosis than acute cholangitis but requires prompt treatment if gangrenous cholecystitis, emphysematous cholecystitis, or torsion of the gallbladder are present. The progression of acute cholecystitis from the mild/moderate to the severe form means the development of the multiple organ dysfunction syndrome (MODS). Organ dysfunction scores, such as Marshall's multiple organ dysfunction (MOD) score, and the sequential organ failure assessment (SOFA) score, are sometimes used to evaluate organ dysfunction in critically ill patients. The Guidelines classify the severity of acute cholecystitis into three grades (Tables 3-5): "severe (grade III)": acute cholecystitis associated with organ dysfunction, "moderate (grade II)": acute cholecystitis associated with difficulty to perform cholecystectomy due to local inflammation, and "mild (grade I)": acute cholecystitis which does not meet the criteria of "severe" or "moderate" acute cholecystitis (these patients have acute cholecystitis but no

Table 3. Criteria for mild (grade I) acute cholecystitis

"Mild (grade I)" acute cholecystitis does not meet the criteria of "severe (grade III)" or "moderate (grade II)" acute cholecystitis. Grade I can also be defined as acute cholecystitis in a healthy patient with no organ dysfunction and only mild inflammatory changes in the gallbladder, making cholecystectomy a safe and low-risk operative procedure.

Table 4. Criteria for moderate (grade II) acute cholecystitis

"Moderate" acute cholecystitis is accompanied by any one of the following conditions:

1. Elevated WBC count $\left(>18000 / \mathrm{mm}^{3}\right)$

2. Palpable tender mass in the right upper abdominal quadrant

3. Duration of complaints $>72 \mathrm{~h}^{\mathrm{a}}$

4. Marked local inflammation (biliary peritonitis, pericholecystic abscess, hepatic abscess, gangrenous cholecystitis, emphysematous cholecystitis)

a Laparoscopic surgery in acute cholecystitis should be performed within $96 \mathrm{~h}$ after the onset (level $2 \mathrm{~b}-4)^{13,14,16}$

Table 5. Criteria for severe (grade III) acute cholecystitis

"Severe" acute cholecystitis is accompanied by dysfunctions in any one of the following organs/systems

1. Cardiovascular dysfunction (hypotension requiring treatment with dopamine $\geqq 5 \mu \mathrm{g} / \mathrm{kg}$ per min, or any dose of dobutamine)

2. Neurological dysfunction (decreased level of consciousness)

3. Respiratory dysfunction $\left(\mathrm{PaO}_{2} / \mathrm{FiO}_{2}\right.$ ratio $\left.<300\right)$

4. Renal dysfunction (oliguria, creatinine $>2.0 \mathrm{mg} / \mathrm{dl}$ )

5. Hepatic dysfunction (PT-INR $>1.5$ )

6. Hematological dysfunction (platelet count $<100000 / \mathrm{mm}^{3}$ ) 
Table 6. Answer pad responses on the criteria for severe (grade III) acute cholecystitis

\begin{tabular}{|c|c|c|c|}
\hline & Agree & $\begin{array}{l}\text { Agree, but needs } \\
\text { minor } \\
\text { modifications }\end{array}$ & Disagree \\
\hline Total $(n=110)$ & $90 \%$ & $10 \%$ & $0 \%$ \\
\hline Panelists from abroad $(n=21)$ & $95 \%$ & $5 \%$ & $0 \%$ \\
\hline Japanese panelists $(n=21)$ & $81 \%$ & $19 \%$ & $0 \%$ \\
\hline Audience $(n=68)$ & $91 \%$ & $9 \%$ & $0 \%$ \\
\hline
\end{tabular}

Table 7. Answer pad responses on the criteria for moderate (grade II) acute cholecystitis

\begin{tabular}{|c|c|c|c|}
\hline & Agree & $\begin{array}{l}\text { Agree, but needs } \\
\text { minor } \\
\text { modifications }\end{array}$ & Disagree \\
\hline Total $(n=109)$ & $78 \%$ & $22 \%$ & $0 \%$ \\
\hline Panelists from abroad $(n=22)$ & $77 \%$ & $23 \%$ & $0 \%$ \\
\hline Japanese panelists $(n=22)$ & $91 \%$ & $9 \%$ & $0 \%$ \\
\hline Audience $(n=65)$ & $74 \%$ & $26 \%$ & $0 \%$ \\
\hline
\end{tabular}

organ dysfunction, and there are mild inflammatory changes in the gallbladder, so that a cholecystectomy can be performed with a low operative risk). Almost unanimous agreement on the criteria was achieved (Tables 6 and 7). When acute cholecystitis is accompanied by acute cholangitis, the criteria for the severity assessment of acute cholangitis should also be taken into account. Being "elderly" per se is not a criterion for severity itself, but indicates a propensity to progress to the severe form, and thus is not included in the criteria for severity assessment.

Acknowledgments. We would like to express our deep gratitude to the Japanese Society for Abdominal Emergency Medicine, the Japan Biliary Association, and the Japanese Society of Hepato-Biliary-Pancreatic Surgery, who provided us with great support and guidance in the preparation of the Guidelines. This process was conducted as part of the Project on the Preparation and Diffusion of Guidelines for the Management of Acute Cholangitis (H-15-Medicine-30), with a research subsidy for fiscal 2003 and 2004 (Integrated Research Project for Assessing Medical Technology), sponsored by the Japanese Ministry of Health, Labour, and Welfare.

We also truly appreciate the panelists who cooperated with and contributed significantly to the International Consensus Meeting held on April 1 and 2, 2006.

\section{References}

1. Juvonen T, Kiviniemi H, Niemela O, Kairaluoma MI. Diagnostic accuracy of ultrasonography and C-reactive protein concentration in acute cholecystitis: a prospective clinical study. Eur J Surg 1992;158:365-9 (level 1b).

2. Ralls PW, Colletti PM, Lapin SA, Chandrasoma P, Boswell WD Jr, Ngo C, et al. Real-time sonography in suspected acute cholecystitis. Prospective evaluation of primary and secondary signs. Radiology 1985;155:767-71 (level 4).

3. Martinez A, Bona X, Velasco M, Martin J. Diagnostic accuracy of ultrasound in acute cholecystitis. Gastrointest Radiol 1986;11: 334-8 (level 4).

4. Ralls PW, Halls J, Lapin SA, Quinn MF, Morris UL, Boswell W. Prospective evaluation of the sonographic Murphy sign in suspected acute cholecystitis. J Clin Ultrasound 1982;10:113-5 (level 4).

5. Bree RL. Further observations on the usefulness of the sonographic Murphy sign in the evaluation of suspected acute cholecystitis. J Clin Ultrasound 1995;23:169-72 (level 4).

6. Hakansson K, Leander P, Ekberg O, Hakansson HO. MR imaging in clinically suspected acute cholecystitis. A comparison with ultrasonography. Acta Radiol 2000;41:322-8 (level 2b).

7. Regan F, Schaefer DC, Smith DP, Petronis JD, Bohlman ME, Magnuson TH. The diagnostic utility of HASTE MRI in the evaluation of acute cholecystitis: half-Fourier acquisition singleshot turbo SE. J Comput Assist Tomogr 1998;22:638-42 (level 4).

8. Shea JA, Berlin JA, Escarce JJ, Clarke JR, Kinosian BP, Cabana $\mathrm{MD}$, et al. Revised estimates of diagnostic test sensitivity and specificity in suspected biliary tract disease. Arch Intern Med 1994;154:2573-81 (level 1b).

9. Ito K, Fujita N, Noda Y, Kobayashi G, Kimura K, Katakura Y, et al. The significance of magnetic resonance cholangiopancreatography in acute cholecystitis (in Japanese with English abstract). Jpn J Gastroenterol 2000;97:1472-9 (level 4). 
10. Fidler J, Paulson EK, Layfield L. CT evaluation of acute cholecystitis: findings and usefulness in diagnosis. Am J Roentgenol 1996;166:1085-8 (level 3b).

11. Mauro MA, McCartney WH, Melmed JR. Hepatobiliary scanning with 99m Tc-PIPIDA in acute cholecystitis. Radiology 1982;142:193-7 (level 4).

12. Bushnell DL, Perlman SB, Wilson MA, Polcyn RE. The rim sign: association with acute cholecystitis. J Nucl Med 1986;27:353-6 (level 4).

13. Brodsky A, Matter I, Sabo E, Cohen A, Abrahamson J, Eldar S. Laparoscopic cholecystectomy for acute cholecystitis: can the need for conversion and the probability of complications be predicted? A prospective study. Surg Endosc 2000;14:755-60 (level $2 b)$.

14. Teixeira JP, Sraiva AC, Cabral AC, Barros H, Reis JR, Teixeira A. Conversion factors in laparoscopic cholecystectomy for acute cholecystitis. Hepatogastroenterology 2000;47:626-30 (level 2b).

15. Halachmi S, DiCastro N, Matter I, Cohen A, Sabo E, Mogilner JG, et al. Laparoscopic cholecystectomy for acute cholecystitis: how do fever and leucocytosis relate to conversion and complications? Eur J Surg 2000;166:136-40 (level 2b)

16. Araujo-Teixeria JP, Rocha-Reis J, Costa-Cabral A, Barros H, Saraiva AC, Araujo-Teixeira AM. Laparoscopic versus open cholecystectomy for cholecystitis (200 cases). Comparison of results and predictive factors for conversion (in French with English abstract). Chirurgie 1999;124:529-35 (level 4).

17. Rattner DW, Ferguson C, Warshaw AL. Factors associated with successful laparoscopic cholecystectomy for acute cholecystitis. Ann Surg 1993;217:233-6 (level 2b).

18. Merriam LT, Kanaan SA, Dawes JG, Angelos P, Prystowsky JB, Rege RV, et al. Gangrenous cholecystitis: analysis of risk factors and experience with laparoscopic cholecystectomy. Surgery 1999;126:680-5 (level 4).

\section{Discussion at the Tokyo International Consensus Meeting}

\section{Diagnostic criteria for acute cholecystitis}

The clinical diagnosis of acute cholecystitis is traditionally based on the patient's clinical presentation, and it is confirmed by the imaging findings. Hence, the initial provisional diagnostic criteria for acute cholecystitis comprised: (1) clinical signs and symptoms, (2) laboratory data, and (3) imaging findings. In the discussion on criteria for "clinical signs and symptoms", $92 \%$ of the Japanese panelists agreed, whereas only $65 \%$ of the panelists from abroad agreed and $4 \%$ disagreed. In regard to the criteria for "laboratory data", $20 \%$ of the
Japanese panelists and $39 \%$ of the panelists from abroad voted "agree, but needs minor modifications". After a discussion among the panelists, several changes were made. In regard to the proposed criteria for "imaging findings", $66 \%-71 \%$ of the Japanese panelists agreed and about $30 \%$ of the panelists voted "agree, but needs minor modifications", and $4 \%$ of the panelists from abroad disagreed, because Tc-HIDA scans were not included. Discussion at the International Consensus Meeting led to the reorganization of these categories as: (1) local signs of inflammation, (2) systemic signs of inflammation, and (3) imaging findings. "Suspected diagnosis" in the provisional criteria was deleted, and two conditions for "definite diagnosis" were established in the final diagnostic criteria. After the discussion, 100\% of the Japanese panelists and $81 \%$ of the panelists from abroad agreed on the final version (refer to Tables 1 and 2; consensus was reached).

\section{Severity assessment criteria for acute cholecystitis}

Concerning criteria for severe (grade III) acute cholecystitis, $81 \%$ of the Japanese panelists and $95 \%$ of the panelists from abroad agreed with the criteria (refer to Tables 5 and 6; consensus was reached). The acute physiology and chronic health evaluation II (APACHE II) score was not included in the assessment criteria, because it is too complicated to apply in community hospitals.

The criteria for moderate (grade II) acute cholecystitis can be defined as acute cholecystitis associated with local inflammatory conditions that make cholecystectomy difficult (Steven Strasberg, USA; Dirk J. Gouma, the Netherlands; Henry Pitt, USA; Sheung-Tat Fan and Joseph W.Y. Lau, Hong Kong; Serafin C. Hilvano, Philippines). On the basis of these aspects, the final criteria for moderate (grade II) acute cholecystitis were defined and were agreed on by $91 \%$ of the Japanese panelists and $77 \%$ of those from abroad (refer to Tables 4 and 7; consensus was reached).

The criteria for mild (grade I) acute cholecystitis were agreed on by approximately $90 \%$ of both the Japanese panelists and the panelists from abroad (consensus was reached). 